\title{
Kerstersia Gyiorum causing Chronic Oti Media: Where a Quinolone Does not Work
}

\author{
Berta MP Vela, Rossi Nuñez, Juan Manuel García-Lechuz, Ana I López-Calleja, Antonio \\ Rezusta, María José Revillo
}

Clinical Microbiology Department, Hospital General Universitario, Miguel Servet, Zaragoza, Spain

Corresponding author: Juan Manuel García-Lechuz, Microbiología, Hospital Miguel Servet, Paseo Isabel La Católica, 1, Zaragoza, Spain, Tel: 34976553790; E-mail: jmgarcialechuz@salud.aragon.es

Received date: May 10, 2017; Accepted date: May 19, 2017, Published date: May 25, 2017

Copyright: (c) 2017 García-Lechuz JM. This is an open-access article distributed under the terms of the Creative Commons Attribution License, which permits unrestricted use, distribution, and reproduction in any medium, provided the original author and source are credited.

Citation: Berta MPV, Rossi N, García-Lechuz JM, López-Calleja Al, Antonio R, et al. Kerstersia Gyiorum causing Chronic Otitis Media: Where a Quinolone Does not Work. J Infec Dis Treat. 2017, 3:1

\section{Abstract}

Chronic otitis media (COM) is an inflammatory disease which affects the mucosal and bone structures of the medium ear, insidiously, slowly progressive, prone to persist and to produce severe sequelae. Staphylococcus aureus following by gram negative bacillus as Proteus spp., Klebsiella spp., Escherichia spp. and Haemophilus influenza are common pathogens causing COM. Recently some cases of COM produced by less known bacteria have been described. This is the case of the genus Kerstersia which has emerged in the literature causing bacteremia and urinary infection, as well as a causative pathogen of chronic otitis.

We have reviewed five cases as well as our own experience with an 88-year-old man transferred to our hospital suffering from persistent otorrhea, finally diagnosed as COM caused by Kerstersia gyiorum resistant to quinolones. Kerstersia genus belongs to the family Alcaligenaceae. A sample from the ear of our patient was taken and gram-negative rods were observed in the Gram stain. After incubation for $24 \mathrm{~h}$, in all media, abundant slightly convex colonies with extended edges, colorful, were isolated and identified by MALDI-TOF (matrixassisted laser desorption ionization time-of-flight) Biotyper 3.1 as Kerstersia gyiorum (score of 2.3. K. gyiorum identification was confirmed by sequencing of the rRNA $16 \mathrm{~S}$ gene and comparing of the sequence obtained with those deposited in GenBank with the NCBI BLASTn algorithm. Our case would be the third case resistant to quinolones reported in the literature.

Keywords: Kerstersia; Chronic otitis; Ciprofloxacin resistant

\section{Introductio}

Chronic otitis media (COM) is an insidious, slowly progressive inflammatory disease affecting the mucosal and bone structures of the medium ear and prone to persist and to produce severe sequelae [1].

Suppurate COM is known as chronic ear discharge through a tympanic drilling, lasting for at least 6 weeks with periods of inactivity [1]. Well-known risk factors for developing a COM are overcrowded living conditions, recurrent respiratory tract infections and smoking [2].

Staphylococcus aureus following by gram negative bacillus as Proteus spp., Klebsiella spp., Escherichia spp. and Haemophilus influenza are common pathogens causing COM. Bacteroides spp. and Fusobacterium spp. are the anaerobes more frequently isolated. There are also mixed infections and several cases of COM produced by less known bacteria as Bordetella, Achromobacter, Alcaligenes and Kerstersia [2-5] have been described.

We presented a case of COM by Kerstersia gyiorum, the third case described resistant to ciprofloxacin.

\section{Case Presentatio}

The case is a male patient, 88 years old, with a history of type 2 diabetes mellitus, hypertension, dyslipidemia, chronic renal failure, chronic heart failure and prostatic hypertrophy. In 2004, he was diagnosed a right tympanic perforation and otorrhea with good clinical outcome after medical treatment.

In May 2016, the patient went to his family doctor complaining of a right otalgia and otorrhea for three weeks and being referred to an ENT specialist. Then, the presence of a foreign body (cotton) in the right ear canal and a tympanic perforation already known was shown. The foreign body was removed and topical corticosteroids (solution dipropionate/ clioquinol beclomethasone; Menaderm eardrops ${ }^{\circledast} 0.25 \mathrm{mg} / 10$ $\mathrm{mg} / \mathrm{ml}, 3$ drops tid, 7 days) were prescribed and monthly reviews were scheduled.

After persistent otorrhea, two regimens of topical ciprofloxacin (Cetraxal ótico $1.2 \mathrm{mg} / 0.4 \mathrm{ml}$ solution ear drops, 3 drops tid, 7 days) are prescribed. So this torpid evolution the patient was again referred to an ENT specialist on July. After his physical exam, a profuse suppuration inside the right ear 
was observed and a sample of the exudate was taken for microbiological studies.

Direct Gram stain showed Gram-positive Cocci and Gramnegative Bacilli but no leukocytes. After incubation for $24 \mathrm{~h}$, a few colonies of Staphylococcus aureus were isolated on Columbia agar (Oxoid, Germany) and Chocolate agar (Chocolate Agar Base, GC Medium, BD DifcoTM, Beckton Dickinson, US). In all media including MacConkey agar (No. 3, Oxoid, Germany), abundant slightly convex colonies with extended edges, colorful from gray hue to clear lavender (Figure 1), oxidase and catalase negative and indol positive were isolated. In the Gram stain from the grown colonies, gram-negative bacilli were observed and then identified by mass spectrometry using the technique of MALDI-TOF (matrixassisted laser desorption ionization time-of-flight) Biotyper 3.1 (Bruker Daltonic GmbH, Bremen, Germany) as Kerstersia gyiorum with a score of 2.1 .

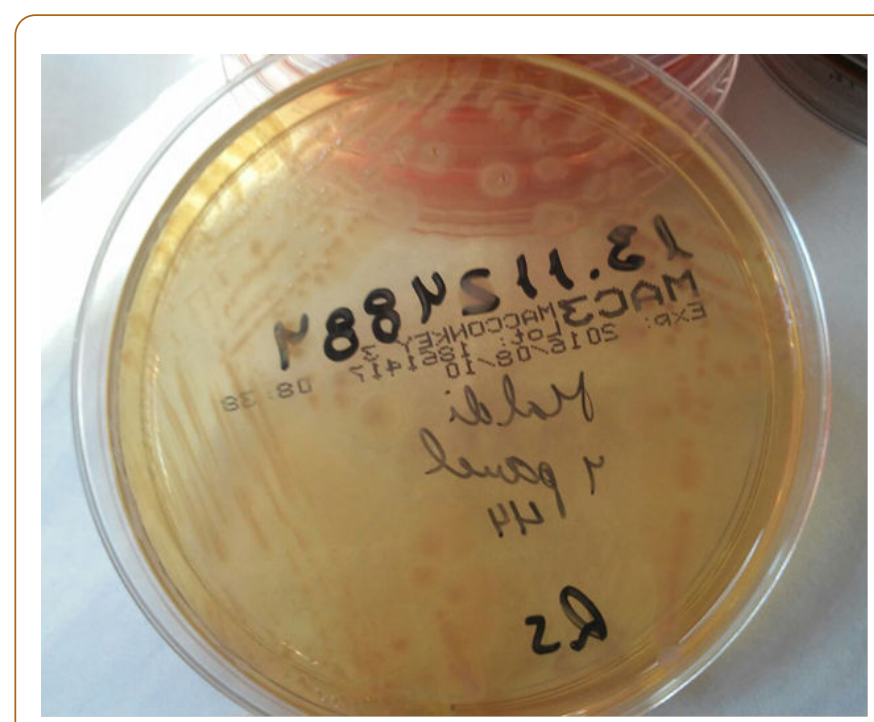

Figure 1 Growing colonies of Kerstesia over blood agar plate and MacConkey agar.

Antibiotic susceptibility test (Table 1) was performed by broth microdilution method using the automated system MicroScan WalkAway ${ }^{\circledast}$ (Siemens Healthcare, Spain currently Beckman Coulter) and the MicroScan ${ }^{\oplus}$ panels (Neg MIC Panel Type 44) were used. The interpretation of the minimum inhibitory concentration (MIC) was performed according to the breakpoints for non-Enterobacteriaceae gram-negative bacilli set by CLSI (Clinical and Laboratory Standards Institute) [6].

Isolates were susceptible to cefotaxime $\leq 1 \mu \mathrm{g} / \mathrm{ml}$, ceftazidime $\leq 1 \mu \mathrm{g} / \mathrm{ml}$, cefepime $\leq 1 \mu \mathrm{g} / \mathrm{ml}$ gentamicin $\leq 2$ $\mu \mathrm{g} / \mathrm{ml}$, tobramycin $\leq 2 \mu \mathrm{g} / \mathrm{ml}$, amikacin $\leq 8 \mu \mathrm{g} / \mathrm{ml}$, piperacillintazobactam $\leq 8 \mu \mathrm{g} / \mathrm{ml}$ tetracycline $\leq 4 \mu \mathrm{g} / \mathrm{ml}$, trimethoprimsulfamethoxazole $\leq 2 / 38 \mathrm{~g} / \mathrm{ml}$, meropenem $\leq 1 \mu \mathrm{g} / \mathrm{ml}$, imipenem $\leq 1 \mu \mathrm{g} / \mathrm{ml}$ and resistant to ciprofloxacin $>2 \mu \mathrm{g} / \mathrm{ml}$ and levofloxacin $>4 \mu \mathrm{g} / \mathrm{ml}$.
K. gyiorum identification was confirmed by sequencing the 16S rRNA gene. The sequence was $99.8 \%$ identical to $K$. gyiorum type strain LMG ...... using the NCBI 16S rRNA gene database.

Table 1 Antibiotic susceptibility profile.

\begin{tabular}{|l|l|l|}
\hline Antibiotic & MIC $(\boldsymbol{\mu g} / \mathbf{m L})$ & Interpretation $^{\mathbf{a}}$ \\
\hline Cefotaxime & $\leq 1$ & $\mathrm{~S}$ \\
\hline Ceftazidime & $\leq 1$ & $\mathrm{~S}$ \\
\hline Cefepima & $\leq 1$ & $\mathrm{~S}$ \\
\hline Gentamicin & $\leq 2$ & $\mathrm{~S}$ \\
\hline Tobramicin & $\leq 4$ & $\mathrm{~S}$ \\
\hline Amikacin & $\leq 8$ & $\mathrm{~S}$ \\
\hline Piperacilin-tazobactam & $\leq 8$ & $\mathrm{~S}$ \\
\hline Trimetoprim-sulfametoxazol & $\leq 2 / 38$ & $\mathrm{~S}$ \\
\hline Meropenem & $\leq 1$ & $\mathrm{~S}$ \\
\hline Imipenem & $\leq 1$ & $\mathrm{~S}$ \\
\hline Ciprofloxacin & $>2$ & $\mathrm{R}$ \\
\hline Levofloxacin & $>4$ & \\
\hline
\end{tabular}

After antibiotic susceptibility report was known, the patient was treated with topical gentamicin (gentamicin/ dexamethasone eye drops solution Colircusi Gentadexa ${ }^{\circledR} 1 / 3$ / $0.5 \mathrm{mg}, 3$ drops tid) plus oral cloxacillin (cloxacillin $500 \mathrm{mg}, 1$ tablet tid) for 7 days. After that, a clinical improvement and otorrhea dry up was clearly observed.

\section{Discussion}

Kerstersia genus belongs to the family Alcaligenaceae. It includes two kinds of species (Kerstersia gyiorum and Kerstersia similis). It is a gram-negative, small $(1-2 \mu \mathrm{m})$, coccobacilli in pairs or short chains. It grows well on standard culture media between 28 and $42^{\circ} \mathrm{C}$. On nutrient agar, the colonies are flat or slightly convex with smooth edges and white to light brown. They are strict aerobes, non- fermenter, catalase positive $[7,8]$. The biochemical features of our strain were similar to those reported in the literature.

K. gyiorum appears as a human pathogen described in the literature reviewed in nine occasions, being isolated from urine samples [9], ulcers [10,11] sputum [12] and causing chronic otitis in five cases [2-5].

The first isolation associated with chronic otitis was reported in 2012 by Almuzara et al. It was in a 16 years-oldmale patient diagnosed with chronic otitis media associated to cholesteatoma [2]. In the other cases $K$. gyiorum has been isolated together with other microorganisms, being difficult to 
determine its pathogenic role attributable solely to $K$. gyiorum

(Table 2).

Table 2 Summary of reported cases of chronic otitis media caused by K. gyioruma.

\begin{tabular}{|c|c|c|c|c|c|c|}
\hline Reference & Age & Background & Isolation in culture & $\begin{array}{l}\text { Ciprofloxacin } \\
(\mathrm{MIC} \mu \mathrm{g} / \mathrm{mL} \text { ) }\end{array}$ & Directed therapy & Outcome \\
\hline [2] & 16 & $\begin{array}{l}\text { - } \quad \mathrm{AOM} \text { and } \\
\text { retroauricular } \\
\text { abscess } \\
\text {-Overcrowding and } \\
\text { unhealthy } \\
\text { conditions }\end{array}$ & Monomicrobial & $1(\mathrm{~S})$ & $\begin{array}{l}\text { Ceftriaxone } 2 \mathrm{~g} \text { iv then } \\
\text { Ciprofloxacin } 500 \mathrm{mg} \text { bid po plus } \\
\text { Amoxicilin/clavulanic, } 1 \mathrm{~g} \text { bid po }\end{array}$ & Favourable \\
\hline [3] & 55 & $\begin{array}{l}\text { - Otorrhoea in } \\
\text { childhood } \\
\text {-Smoking } \\
\text {-Bilateral mastoid } \\
\text { surgery }\end{array}$ & $\begin{array}{l}\text { Polymicrobial } \\
\text { Corynebacterium } \\
\text { amycolatum }\end{array}$ & $>32(\mathrm{R})$ & Trimetoprim/sulfametoxazol & Favourable \\
\hline [4] & 53 & $\begin{array}{l}\text { - Chronic otorrhea } \\
\text { in adulthood } \\
\text {-Smoking }\end{array}$ & $\begin{array}{l}\text { Polymicrobial } \\
\text { Proteus mirabilis }\end{array}$ & $1(\mathrm{l})$ & Topical ciprofloxacin & Favourable \\
\hline [4] & 33 & $\begin{array}{l}\text { - Chronic otorrhea } \\
\text { in adulthood }\end{array}$ & $\begin{array}{l}\text { Polymicrobial } \\
\text { Staphylococcus aureus } \\
\text { E. coli }\end{array}$ & $1(\mathrm{l})$ & Topical ciprofloxacino & Favourable \\
\hline [5] & 25 & $\begin{array}{l}\text { - Otorrhoea } \\
\text { In childhood }\end{array}$ & $\begin{array}{l}\text { Polymicrobial } \\
\text { Pseudomonas } \\
\text { aeruginosa }\end{array}$ & $>2(\mathrm{R})$ & $\begin{array}{l}\text { Imipenem } 500 \text { mg/qid iv, } \\
10 \text { days }\end{array}$ & Favourable \\
\hline Present Case & 88 & $\begin{array}{l}\text { - Chronic otorrhea } \\
\text { in adulthood } \\
\text { - Tympanum } \\
\text { drilling }\end{array}$ & $\begin{array}{l}\text { Polymicrobial } \\
\text { Staphylococcus aureus }\end{array}$ & $>2(\mathrm{R})$ & $\begin{array}{l}\text { Topical gentamicin } \\
\text { Cloxacilin po } 500 \mathrm{mg} \text { tid } \\
7 \text { days }\end{array}$ & Favourable \\
\hline
\end{tabular}

In our case, Staphylococcus aureus was also isolated however we considered Kerstersia gyiorum as a true pathogen in view of the chronicity of the disease, the poor response to treatment with ciprofloxacin, the observation of bacilli in the Gram stain and, the abundant growth into the inoculated plates. The cases reported so far in literature, were patients with chronic ear disease, as our patient, so it is deductible $K$. gyiorum has a pathogenic role in these patients. In our case there was no history of smoking or overcrowded conditions, described by other authors [2-4].

Currently, the identification of new species by MALDI-TOF and $16 \mathrm{~S}$ rRNA sequencing are essential for microbiological diagnosis.

There are no cutoffs of antibiotic susceptibility in CLSI or EUCAST (European Committee on Antimicrobial Susceptibility Testing) specific to this genus. We used the CLSI criteria for non-Enterobacteriaceae.

According to Harris et al. [13] about the use of quinolones and aminoglycosides in the treatment of chronic suppurative otitis media, quinolones are bactericidal allowing different routes of administration and therefore outpatient treatments. They also are less ototoxic than aminoglycosides and have the same efficacy in resolving otorrhea all of which makes them a first-line option in chronic otitis, especially in cases with tympanic perforation.
Kerstersia isolates are generally susceptible to ciprofloxacin and cefotaxime [8] although recently there have been described two strains resistant to ciprofloxacin $[3,5]$. Treatment failure is considered when otorrhea persists approximately three weeks after medical therapy. Causes of failure include the presence of resistant microorganisms, the presence of cholesteatoma or a poor adherence to treatment, being therefore necessary a microbiological analysis including an antibiotic susceptibility study [14].

Our case would be the third case resistant to ciprofloxacin reported in the literature. The prior long treatment with ciprofloxacin may be the clue of further selection of resistant strains.

In summary, $K$. gyiorum is therefore a novel pathogen to be considered in the differential diagnosis of microorganisms causing chronic otitis media.

Microbiological diagnosis is the important key to guide the antimicrobial treatment because of the lack of homogeneity in its antibiotic susceptibility.

\section{Conflic of Interest}

The authors declare not to have an association that might pose a conflict of interest. 
There is no fund, non-financial support received by the authors of the manuscript.

\section{References}

1. Mittal R, Lisi CV, Gerring R, Mittal J, Mathee K, et al. (2015) Current concepts in the pathogenesis and treatment of chronic suppurative otitis media. J Med Microbiol 64: 1103-1106.

2. Almuzara MN, Barberis CM, Traglia GM, Ordonez AM, Famiglietti AMR, et al. (2012) Isolation of Kerstersia gyiorum from a Patient with Cholesteatomatous Chronic Otitis Media. J Clin Microbiol 50: 3809-3811.

3. Pence MA, Sharon J, Elvania TE, Pakalniskis BL (2013) Two Cases of Kerstersia gyiorum Isolated from Sites of Chronic Infection. J Clin Microbiol 51: 2001-2004.

4. Mwalutende A, Mshana SE, Mirambo MM, Mushi MF, Chalya PL, et al. (2014) Two cases of chronic suppurative otitis media caused by Kerstersia gyiorum in Tanzania: is it an underappreciated pathogen in chronic otitis media. Int J Infect Dis 29: 251-253.

5. Uysal EB, Celik C, Tuzcu N, Can F, Dogan M, et al. (2015) A case of chronic suppurative otitis media caused by Kerstersia gyiorum. APMIS 123: 986-989.

6. CLSI (2016) Performance standards for antimicrobial susceptibility testing 26th ed. CLSI supplement M100S. Clinical and Laboratory Standards Institute, Wayne, PA,USA.
7. Wirsing C, Riffelmann M, Coenye TB, Pfaller MA, Richter SS (2015) Manual of Clinical Microbiology 11th ed. Washington DC. American Society of Microbiology 838-850.

8. Coenye T (2003) Kerstersia gyiorum gen. nov., sp. nov., a novel Alcaligenes faecalis-like organism isolated from human clinical samples, and reclassification of Alcaligenes denitrificans Ruger and Tan 1983 as Achromobacter denitrificans comb. nov. Int J Syst Evol Microbiol 53: 1825-1831.

9. Ogawa Y, Lee ST, Kasahara K, Koizumi A, Chihara Y, et al. (2016) A first case of isolation of Kerstersia gyiorum from urinary tract. J Infect Chemother 22: 265-267.

10. Greninger $\mathrm{AL}$, Kozyreva $\mathrm{V}$, Truong $\mathrm{CL}$, Longoria $\mathrm{R}$, Chaturvedi $\mathrm{V}$ (2015) Draft Genome Sequence of Kerstersia gyiorum CG1, Isolated from a Leg Ulcer. Genome Announc 3: e01036-15.

11. Bostwick AD, Zhang C, Manninen K, Touchberry J, Greene SR, et al. (2015) Bacteremia Caused by Kerstersia gyiorum. J Clin Microbiol 53: 1965-1967.

12. Deutscher M, Severing J, Balada L (2014) Kerstersia gyiorum Isolated from a Bronchoalveolar Lavage in a Patient with a Chronic Tracheostomy. Case Rep Infect Dis 1-3.

13. Harris AS, Elhassan HA, Flook EP (2016) why are ototopical aminoglycosides still first-line therapy for chronic suppurative otitis media? A systematic review and discussion of aminoglycosides versus quinolones. J Laryngol Otol 130: 2-7.

14. Karov I (1996) Preoperative treatment of children with chronic suppurative otitis media. Folia Med (Plovdiv) 38: 63-69. 